\title{
On weighted path lengths and distances in increasing trees
}

\author{
M. Kuba and A. Panholzer \\ Institut für Diskrete Mathematik und Geometrie \\ Technische Universität Wien \\ Wiedner Hauptstr. 8-10/104 \\ 1040 Wien, Austria, \\ Markus.Kuba@tuwien.ac.at, Alois.Panholzer@tuwien.ac.at \\ Corresponding Author: M. Kuba
}

November 23, 2006 


\begin{abstract}
We study weighted path lengths (depths) and distances for increasing tree families. For those subclasses of increasing tree families, which can be constructed via an insertion process, e.g., recursive trees, plane-oriented recursive trees and binary increasing trees, we can determine the limiting distribution which can be characterized as a generalized Dickman's infinitely divisible distribution.
\end{abstract}

Keywords: Increasing trees, Depths, Distances, Infinitely divisible distribution, Limiting distribution

2000 Mathematics Subject Classification Primary 05C05, 60C05, Secondary 60F05. 


\section{Introduction}

The analysis of the length of paths in tree families has received a lot of attention, see, e.g., $[4,7,11]$, often due to their importance in the analysis of algorithms. Recently Aguech, Lasmar and Mahmoud [1] introduced weighted path lengths in binary search trees. They have shown that different limit laws arise for weighted path lengths depending on the label of the investigated nodes. In particular they have derived the limit law for the weighted path length to the minimal label, which is Dickman's infinitely divisible distribution.

We extend the studies of weighted path lengths to other important labeled trees, namely to families of increasing trees. We will also consider weighted distances in increasing trees, where for both parameters we will encounter a generalized Dickman distribution as the limit law. In order to obtain the limiting distribution results of the parameters considered we will use suitable distributional decompositions of the (ordinary) path lengths and distances, which will be extended to corresponding decompositions of their weighted counterparts. Previous results concerning ordinary depths (path lengths) and distances for increasing tree families can be found in Dobrow [5], Dobrow and Smythe [6] and Kuba and Panholzer [16]. The distributional decomposition of the weighted parameters and an application of Curtiss' theorem leads to limiting distribution results for the random variables considered. The arguments used in the present paper are related to Hwang and Tsai [8], where the limit law of the algorithm Quickselect was obtained with a similar technique.

Increasing trees are labeled trees where the nodes of a tree of size $n$ are labeled by distinct integers of the set $\{1, \ldots, n\}$ in such a way that each sequence of labels along any branch starting at the root is increasing. We are concerned throughout this paper with certain combinatorial models of increasing trees, called simple families of increasing trees. One can consider them as increasingly labeled simply generated trees (simply generated trees have been introduced by Meir and Moon [15]). A formal description of simple families of increasing trees is given in Subsection 2.1. A thorough study of simple families (= varieties) of increasing trees was conducted in Bergeron et. al. [2].

The interest in simple families of increasing trees stems from the fact that several important tree models, as, e.g., recursive trees, plane-oriented recursive trees and binary increasing trees (also called tournament trees), are special instances of simple families of increasing trees. These tree models are of importance in many applications (see the survey paper of Mahmoud and Smythe [14] and also [13]), e.g., they are used to describe the spread of epidemics, for pyramid schemes, and they are used as a sim- 
plified growth model of the world wide web: plane-oriented recursive trees are a special instance of the so called Albert-Barabási model for scale-free networks (see e. g. [3]).

The tree families mentioned above (i.e., recursive trees, plane-oriented recursive trees and binary increasing trees) can be described not only combinatorially, but also in a probabilistic way via a tree evolution process. This means that for every tree $T^{\prime}$ of size $n$ with vertices $v_{1}, \ldots, v_{n}$ one can give probabilities $p_{T^{\prime}}\left(v_{1}\right), \ldots, p_{T^{\prime}}\left(v_{n}\right)$, such that when starting with a random tree $T^{\prime}$ of size $n$ of the tree family considered, choosing a vertex $v_{i}$ in $T^{\prime}$ according to the probabilities $p_{T^{\prime}}\left(v_{i}\right)$ and attaching node $n+1$ to it, one obtains again a random tree $T$ of size $n+1$ of the tree family considered. See, e.g., [14] for such a description of recursive trees and plane-oriented recursive trees. However, it has been shown in [17] that it is not possible to describe every simple family of increasing trees as defined in Subsection 2.1 via a tree evolution process. Moreover, a full characterization of those simple families of increasing trees having this additional property has been given there. This characterization is restated in Subsection 2.2. Throughout this paper we will choose the term grown simple families of increasing trees for the subclass of increasing tree families, which can be generated by a tree evolution process. We will restrict ourselves to the study of this subclass of simple families of increasing trees.

In a rooted tree the depth of node $v$, also called the level of node $v$ or path length from $v$ to the root, is measured by the number of edges lying on the unique path from the root to node $v$. For labeled rooted trees we consider a generalization of the depth. Let $W_{n, j}$ denote the weighted depth (or weighted path length) of node $j$ in a size $n \geq j$ random grown simple increasing tree, which is the sum of the labels of the nodes encountered on the path from $j$ to the root labeled 1 , where $W_{1,1}=1$ and $W_{n, j} \geq j+1$. For instance if the nodes labeled $\lambda_{1}, \ldots, \lambda_{k}$, with $1=\lambda_{1}<\lambda_{2}<\cdots<\lambda_{k}=j$, are visited on the path from the root to $j$ then the weighted depth equals $\sum_{l=1}^{k} \lambda_{k}$.

Further we consider the weighted distance $\mathcal{W}_{n, j}$ between node $j$ and node $n$ in a size $n$ random grown simple increasing tree which is the sum of the labels of the nodes encountered on the path from $n$ to the node labeled $j$ (hence $\mathcal{W}_{n, 1}=W_{n, n}$ ).

In the tree of Figure 1 we have, e.g, $W_{9,1}=1, W_{9,2}=3, W_{9,3}=4, W_{9,4}=8$, $W_{9,5}=6, W_{9,6}=12, W_{9,7}=8, W_{9,8}=20$ and $W_{9,9}=17$ and $\mathcal{W}_{9,4}=24, \mathcal{W}_{9,8}=36$.

We will show that $W_{n, j}$ and $\mathcal{W}_{n, j}$, appropriately scaled, lead to generalized Dickman distributions.

Related results for binary search trees are due to Aguech, Lasmar and Mahmoud [1]. Further we refer to the limit law of Quickselect, see Mahmoud, Modarres and Smythe [12] and Hwang and Tsai [8], which is also Dickman's infinitely divisible distribution.

We denote with $X \stackrel{(d)}{=} Y$ the equality in distribution of the random variables $X$ and 


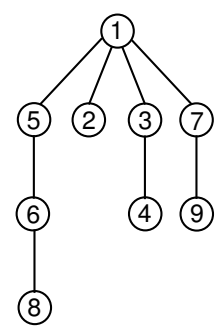

Figure 1: A size 9 recursive tree.

$Y$, and with $X_{n} \stackrel{(d)}{\longrightarrow} X$ the weak convergence, i.e., the convergence in distribution, of the sequence of random variables $X_{n}$ to a random variable $X$. For independent random variables $X$ and $Y$ we write $X \oplus Y$ for the sum of $X$ and $Y$. For not necessarily mutually independent random variables $X$ and $Y$ we write $X+Y$. We denote with $H_{n}:=\sum_{k=1}^{n} \frac{1}{k}$ the $n$-th harmonic number and with $H_{n}^{(a)}:=\sum_{k=1}^{n} \frac{1}{k^{a}}$ the $n$-th harmonic number of order $a$. We denote with $\mathbb{I}\left(\mathcal{A}_{k}\right)$ the indicator of the event $\mathcal{A}_{k}$.

In the next section we gather some results about increasing tree families and the generalized Dickman distribution. In Sections 3 and 4 we state the main limiting distribution results of this work concerning the weighted depth and the weighted distance. Section 5 is devoted to the derivation of the distribution laws of the weighted depths and distances. The last section contains the proofs of the limiting distribution results.

\section{Preliminaries}

\subsection{Combinatorial description of increasing tree families}

Formally, a class $\mathcal{T}$ of a simple family of increasing trees can be defined in the following way. We start with a sequence of non-negative numbers $\left(\varphi_{k}\right)_{k \geq 0}$ with $\varphi_{0}>0$. The sequence $\left(\varphi_{k}\right)_{k \geq 0}$ is called the degree-weight sequence. We assume that there exists a $k \geq 2$ with $\varphi_{k}>0$. The degree-weight sequence is used to define the weight $w(T)$ of any ordered tree $T$ by $w(T):=\prod_{v} \varphi_{d(v)}$, where $v$ ranges over all vertices of $T$ and $d(v)$ is the out-degree of $v$. Furthermore, $\mathcal{L}(T)$ denotes the set of different increasing labelings of the tree $T$ with distinct integers $\{1,2, \ldots,|T|\}$, where $|T|$ denotes the size of the tree $T$, and $L(T):=|\mathcal{L}(T)|$ its cardinality. Then the family $\mathcal{T}$ consists of all trees $T$ together with their weights $w(T)$ and the set of increasing labelings $\mathcal{L}(T)$.

For a given degree-weight sequence $\left(\varphi_{k}\right)_{k \geq 0}$ with a degree-weight generating function 
$\varphi(t):=\sum_{k \geq 0} \varphi_{k} t^{k}$, we define now the total weights by $T_{n}:=\sum_{|T|=n} w(T) \cdot L(T)$. It follows then that the exponential generating function $T(z):=\sum_{n \geq 1} T_{n} \frac{z^{n}}{n !}$ satisfies the autonomous first order differential equation

$$
T^{\prime}(z)=\varphi(T(z)), \quad T(0)=0 .
$$

Remark 1. The formal definition of simple families of increasing trees is similar to the definition of simply generated trees, which are used as an underlying tree model. Note that simply generated trees are unlabeled trees, whereas simple families of increasing trees are increasingly labeled.

\subsection{Characterization of grown simple families of increasing trees}

It turned out that a special subclass of simple families of increasing trees can be constructed via a tree evolution process. We will describe now the characterization of grown simple increasing tree families via the degree-weight generating function $\varphi(t)$ as obtained in [17].

Lemma 1 ([17]). A simple family of increasing trees $\mathcal{T}$ can be constructed via a tree evolution process and is thus a grown simple family of increasing trees iff the degreeweight generating function $\varphi(t)=\sum_{k \geq 0} \varphi_{k} t^{k}$ is given by one of the following three formula (with constants $c_{1}, c_{2} \in \mathbb{R}$ ).

Case $A$ (recursive trees):

$$
\varphi(t)=\varphi_{0} e^{\frac{c_{1} t}{\varphi_{0}}}, \text { for } \varphi_{0}>0, c_{1}>0, \quad\left(\text { defining } c_{2}:=0\right),
$$

Case $B$ (d-ary trees):

$$
\varphi(t)=\varphi_{0}\left(1+\frac{c_{2} t}{\varphi_{0}}\right)^{d}, \text { for } \varphi_{0}>0, c_{2}>0, d:=\frac{c_{1}}{c_{2}}+1 \in\{2,3,4, \ldots\},
$$

Case $C$ (generalized plane-oriented recursive trees):

$$
\varphi(t)=\frac{\varphi_{0}}{\left(1+\frac{c_{2} t}{\varphi_{0}}\right)^{-\frac{c_{1}}{c_{2}}-1}}, \text { for } \varphi_{0}>0,0<-c_{2}<c_{1} .
$$

The tree evolution process which generates random trees of arbitrary size $n$ is for grown simple families of increasing trees given as follows:

- Step 1: The process starts with the root labeled by 1. 
- Step $i+1$ : At step $i+1$ the node with label $i+1$ is attached to any previous node $v$ (with out-degree $d(v)$ ) of the already grown tree of size $i$ with probabilities $p(v)$ given by:

$$
p(v)= \begin{cases}\frac{1}{i}, & \text { for Case } A, \\ \frac{d-d(v)}{(d-1) i+1}, & \text { for Case B, } \\ \frac{d(v)+\alpha}{(\alpha+1) i-1}, \quad \text { with } \alpha:=-1-\frac{c_{1}}{c_{2}}>0, & \text { for Case } C .\end{cases}
$$

The constants $c_{1}, c_{2}$ appearing in Lemma 1 come from an equivalent characterization of grown simple families of increasing trees. The total weights $T_{n}$ of trees of size $n$ of $\mathcal{T}$ satisfy for all $n \in \mathbb{N}$ the equation

$$
\frac{T_{n+1}}{T_{n}}=c_{1} n+c_{2} .
$$

For grown simple increasing tree families the constants $c_{1}$ and $c_{2}$ describe the growth of the total weights from a tree of size $n$ to a tree of size $n+1$.

$$
T_{n}=\varphi_{0} \prod_{k=1}^{n-1}\left(c_{1} k+c_{2}\right)=\varphi_{0} c_{1}^{n-1}(n-1) !\left(\begin{array}{c}
n-1+\frac{c_{2}}{c_{2}} \\
n-1
\end{array}\right) .
$$

For recursive trees we set $c_{2}=0$ and for $d$-ary increasing trees $d=\frac{c_{1}}{c_{2}}+1$.

Since grown simple increasing trees with degree-weight generating function $\varphi(t)$ are a subclass of simple families of increasing trees, the generating function $T(z)$ of the total weights also satisfies the differential equation (1).

Solving either the differential equation (1) or using (2) one obtains the following explicit formulæ for the exponential generating function $T(z)$ :

$$
T(z)= \begin{cases}\frac{\varphi_{0}}{c_{1}} \log \left(\frac{1}{1-c_{1} z}\right), & \text { Case A } \\ \frac{\varphi_{0}}{c_{2}}\left(\frac{1}{\left(1-(d-1) c_{2} z\right)^{\frac{1}{d-1}}}-1\right), & \text { Case B } \\ \frac{\varphi_{0}}{c_{2}}\left(\frac{1}{\left(1-c_{1} z\right)^{\frac{c_{2}}{c_{1}}}}-1\right), & \text { Case C. }\end{cases}
$$




\subsection{The Dickman distribution and generalizations}

The Dickman function $\rho(u)$, which appears in analytic number theory, is defined as the continuous solution of the differential-difference equation

$$
u \rho^{\prime}(u)+\rho(u-1)=0 \quad(u>1),
$$

with initial condition $\rho(u)=1$ for $0 \leq u \leq 1$ and with $\rho(u)$ differentiable on $(1, \infty)$. It is known that the Dickman function is positive and decreasing over the entire interval $(1, \infty)$. Note that

$$
\int_{0}^{\infty} \rho(v) d v=e^{\gamma}
$$

where $\gamma$ denotes Euler's constant. For simplicity of reference, we call the distribution with the density function $e^{-\gamma} \rho(v)$ the Dickman distribution.

Penrose and Wade introduced in [18] the generalized Dickman distribution. Given $\theta>0$, a random variable $X$ has a generalized Dickman distribution with shape parameter $\theta$, or

$$
X \stackrel{(d)}{=} \mathrm{GD}(\theta)
$$

if it satisfies the distributional fixed-point identity

$$
X \stackrel{(d)}{=} \mathrm{U}^{1 / \theta}(1+X)
$$

where $\mathrm{U}$ is uniform on $(0,1]$ and is independent of the $X$ on the right. Some other known properties of the generalized Dickman distribution stated in [18] are listed as follows.

- If $X \stackrel{(d)}{=} \mathrm{GD}(\theta)$, then the Laplace transform of $X$ is given by

$$
\psi(t)=\mathbb{E}\left(e^{-t X}\right)=\exp \left(\theta \int_{0}^{t} \frac{e^{-s}-1}{s} d s\right), \quad t \in \mathbb{R} .
$$

- The $\operatorname{GD}(\theta)$ distribution is infinitely divisible.

- If $X \stackrel{(d)}{=} \mathrm{GD}(\theta)$, then the moments $\mathbb{E}\left(X^{k}\right)$ satisfy $\mathbb{E}\left(X^{0}\right)=1$ and for integer $k \geq 1$

$$
\mathbb{E}\left(X^{k}\right)=\frac{\theta}{k} \sum_{j=0}^{k-1}\left(\begin{array}{l}
k \\
j
\end{array}\right) \mathbb{E}\left(X^{j}\right) .
$$

For $\theta=1$ the $\operatorname{GD}(\theta)$ distribution is just the ordinary Dickman distribution. For more properties of the $\operatorname{GD}(\theta)$ distribution see Penrose and Wade [18].

Now we state the main results of this paper, the limit laws of the weighted depths and distances. 


\section{Results for weighted depths}

\subsection{Limit distribution results}

Theorem 1. The limiting distribution of the random variable $\frac{W_{n, j}-j}{j}$ is a generalized Dickman distribution with parameter $\theta=1+\frac{c_{2}}{c_{1}}>0$.

$$
\lim _{j \rightarrow \infty} \frac{W_{n, j}-j}{j} \stackrel{(d)}{=} \mathrm{GD}\left(1+\frac{c_{2}}{c_{1}}\right)
$$

or equivalently let $\psi_{j}(t):=\mathbb{E}\left(e^{-t\left(W_{n, j}-j\right)}\right)$, then

$$
\lim _{j \rightarrow \infty} \psi_{j}\left(\frac{t}{j}\right)=\exp \left(\left(1+\frac{c_{2}}{c_{1}}\right) \int_{0}^{t} \frac{e^{-v}-1}{v} d v\right)
$$

for constants $c_{1}, c_{2}$ as in (2).

Remark 2. Note that the random variable $W_{n, j}$ is independent of $n$ and only depends on $j: W_{n, j} \stackrel{(d)}{=} W_{j, j}$. Therefore we consider the limit $j \rightarrow \infty$.

For the readers convenience we separately present the limit laws for the most important subfamilies of grown simple increasing trees.

Corollary 1. For the three most prominent tree families we obtain the following result.

- Recursive Trees $\left(c_{1}=1, c_{2}=0\right)$ : The limit distribution of $\frac{W_{n, j}-j}{j}$ is Dickman, $\mathrm{GD}(1)$.

$$
\lim _{j \rightarrow \infty} \mathbb{P}\left\{\frac{W_{n, j}-j}{j} \leq x\right\}=e^{-\gamma} \int_{0}^{x} \rho(v) d v, \quad x>0 .
$$

- Binary Increasing trees $\left(c_{1}=c_{2}=1\right)$ : The limit distribution of $\frac{W_{n, j}-j}{j}$ is the convolution of two Dickman distributions, GD(2).

$$
\lim _{j \rightarrow \infty} \mathbb{P}\left\{\frac{W_{n, j}-j}{j} \leq x\right\}=e^{-2 \gamma} \int_{0}^{x} \rho(v) \rho(x-v) d v, \quad x>0 .
$$

- Plane oriented recursive trees $\left(c_{1}=2, c_{2}=-1\right)$ : The limit distribution of $\frac{W_{n, j}-j}{j}$ a generalized Dickman distribution $\mathrm{GD}(\theta)$ with parameter $\theta=\frac{1}{2}$.

Next we are going to state the main results for weighted distances. 


\section{Results for weighted distances}

\subsection{Limit distribution results}

Theorem 2. For fixed $j$ and $n \rightarrow \infty$ the limiting distribution of the weighted distance $\mathcal{W}_{n, j}$ between node $j$ and node $n$ in a size $n$ grown simple increasing tree is a generalized Dickman distribution with parameter $\theta=1+\frac{c_{2}}{c_{1}}>0$.

$$
\lim _{n \rightarrow \infty} \frac{\mathcal{W}_{n, j}-n}{n} \stackrel{(d)}{=} \mathrm{GD}\left(1+\frac{c_{2}}{c_{1}}\right)
$$

or equivalently $\mathbb{E}\left(e^{-t\left(\mathcal{W}_{n, j}-n\right)}\right)=\psi_{n}(t)$.

$$
\lim _{j \rightarrow \infty} \psi_{n}\left(\frac{t}{n}\right)=\exp \left(\left(1+\frac{c_{2}}{c_{1}}\right) \int_{0}^{t} \frac{e^{-v}-1}{v} d v\right)
$$

for constants $c_{1}, c_{2}$ as in (2).

Theorem 3. The limiting distribution of the weighted distance $\mathcal{W}_{n, j}$ between node $j$ and node $n$ in a grown simple families of increasing tree satisfying $c_{1}=-2 c_{2}$ depends on the growth of $j$.

- The region $j=o(n)$ : The limiting distribution is a generalized Dickman distribution with parameter $\theta=1 / 2$. Let $\mathbb{E}\left(e^{-t\left(\mathcal{W}_{n, j}-n-j\right)}\right)=\psi_{n}(t)$, then

$$
\lim _{n \rightarrow \infty} \psi_{n}\left(\frac{t}{n}\right)=\exp \left(\frac{1}{2} \int_{0}^{t} \frac{e^{-v}-1}{v} d v\right)
$$

- The region $j=\mu n$, with $0<\mu<1$. The limiting distribution can be characterized via its Laplace transform. Let $\mathbb{E}\left(e^{-t\left(\mathcal{W}_{n, j}-n-j\right)}\right)=\psi_{n}(t)$.

$$
\lim _{n \rightarrow \infty} \psi_{n}\left(\frac{t}{n}\right)=\exp \left(\frac{1}{2} \int_{0}^{\mu t} \frac{e^{-v}-1}{v} d v+\int_{\mu t}^{t} \frac{e^{-v}-1}{v} d v\right),
$$

- The region $n-j=o(n)$ : The limiting distribution is a Dickman distribution.

$$
\lim _{n \rightarrow \infty} \mathbb{P}\left\{\frac{\mathcal{W}_{n, j}-j-n}{n} \leq x\right\}=e^{-\gamma} \int_{0}^{x} \rho(v) d v, \quad x>0
$$


Remark 3. Basically Theorem 2 just tells that for fixed $j$ the random variable $\mathcal{W}_{n, j}$ behaves as the weighted depth $W_{n, 1}$.

Unfortunately we are not able to describe the limiting distribution for the full range of $j$ for the all grown simple families of increasing trees. E. g. up to now we are not able to determine the weighted distance between nodes $n-1$ and $n$ for all grown simple families of increasing trees. We can only obtain the limit law for the subfamily admitting $c_{1}=-2 c_{2}$ (containing plane oriented recursive trees), due to the specific decomposition of the underlying ordinary distance for this subfamily.

\section{Deriving the distribution law}

We will derive distributional decompositions for the weighted depths and distances by using the underlying decompositions of the ordinary depths and distances.

\subsection{Weighted Depth}

Dobrow and Smythe [6] already provided a characterization of the ordinary depth, which can be adapted for describing the weighted depth in grown simple families of increasing trees (see also [17] and [16]).

Lemma 2 (Dobrow \& Smythe). The ordinary depth of node $j$ in a size $n \geq j$ random grown simple increasing tree $D_{n, j}$ admits the following distribution law.

$$
D_{n, j} \stackrel{(d)}{=} D_{j, j} \stackrel{(d)}{=} \bigoplus_{k=1}^{j-1} B_{k},
$$

where $B_{k} \stackrel{(d)}{=} \operatorname{Be}\left(p_{k}\right)$ with $p_{k}=\frac{1+\frac{c_{2}}{c_{1}}}{k+\frac{c_{2}}{c_{1}}}$ for $1 \leq k \leq j-1$. Note that $B_{k} \stackrel{(d)}{=} \operatorname{Be}\left(p_{k}\right) \stackrel{(d)}{=} \mathbb{I}\left(A_{k}\right)$, where $A_{k}$ denotes the event that node $k$ is on the path from the root to node $j$. Further the $B_{k}$ 's are independent.

As an immediate consequence of Lemma 2 we obtain the distribution law of the weighted depth $W_{n, j}$, by simply multiplying all the indicators $\mathbb{I}\left(A_{k}\right)$ with $k$. This corresponds to counting the labels of all nodes on the unique path from $j$ to the root.

Proposition 1. The weighted depth of node $j$ in a size $n \geq j$ random grown simple increasing tree $W_{n, j}$ admits the following distribution law.

$$
W_{n, j} \stackrel{(d)}{=} W_{j, j} \stackrel{(d)}{=} j \oplus \bigoplus_{k=1}^{j-1} \mathcal{B}_{k},
$$


where $\mathcal{B}_{k}=\mathcal{B}_{k}\left(c_{1}, c_{2}\right)$ are a sequence of of independent random variables such that

$$
\mathbb{P}\left(\mathcal{B}_{k}=k\right)=\frac{1+\frac{c_{2}}{c_{1}}}{k+\frac{c_{2}}{c_{1}}} \quad \text { and } \quad \mathbb{P}\left(\mathcal{B}_{k}=0\right)=1-\frac{1+\frac{c_{2}}{c_{1}}}{k+\frac{c_{2}}{c_{1}}} .
$$

Note that $\mathcal{B}_{k} \stackrel{(d)}{=} k \cdot \mathbb{I}\left(A_{k}\right)$, where again $A_{k}$ denotes the event that node $k$ is on the path from the root to node $j$. Further the $\mathcal{B}_{k}$ 's are independent.

We also get as a byproduct the expectation and variance of $W_{n, j}$

Corollary 2. The expectation and the variance of the random variable $W_{n, j}$ are given as follows.

$$
\begin{aligned}
\mathbb{E}\left(W_{n, j}\right)= & \left(1+\frac{c_{2}}{c_{1}}\right)(j-1)-\frac{c_{2}}{c_{1}}\left(1+\frac{c_{2}}{c_{1}}\right)\left(H_{j-1+\frac{c_{2}}{c_{1}}}-H_{\frac{c_{2}}{c_{1}}}\right), \\
\mathbb{V}\left(W_{n, j}\right)= & \frac{1+\frac{c_{2}}{c_{1}}}{2} j(j-1)-\left(1+\frac{c_{2}}{c_{1}}\right)\left(1+2 \frac{c_{2}}{c_{1}}\right)(j-1) \\
& +\left(1+\frac{c_{2}}{c_{1}}\right) \frac{c_{2}}{c_{1}}\left(2+3 \frac{c_{2}}{c_{1}}\right)\left(H_{j-1+\frac{c_{2}}{c_{1}}}-H_{\frac{c_{2}}{c_{1}}}\right)-\frac{c_{2}^{2}}{c_{1}^{2}}\left(1+\frac{c_{2}}{c_{1}}\right)^{2}\left(H_{j-1+\frac{c_{2}}{c_{1}}}^{(2)}-H_{\frac{c_{2}}{c_{1}}}^{(2)}\right) .
\end{aligned}
$$

\subsection{Weighted Distance}

We will use the description for the ordinary distance $\Delta_{n, j}$ between $n$ and $j$ to derive the distribution for the weighted distance $\mathcal{W}_{n, j}$. Dobrow and Smythe provided the distribution law of the (ordinary) distance in [6].

Lemma 3 (Dobrow \& Smythe). The distance of between node $j$ and node $n$ in a random grown simple increasing tree $\Delta_{n, j}$ admits the following distribution law.

$$
\Delta_{n, j} \stackrel{(d)}{=} \Delta_{j+1, j} \oplus \bigoplus_{k=j+1}^{n-1} B_{k}
$$

where $B_{k} \stackrel{(d)}{=} \mathrm{Be}\left(p_{k}\right)$ with $p_{k}=\frac{1+\frac{c_{2}}{c_{1}}}{k+\frac{c_{2}}{c_{1}}}$ for $j+1 \leq k \leq n-1$.

Remark 4. The Bernoulli random variables $B_{k}$, appearing in Lemma 3, which characterize the distribution law of $\Delta_{n, j}$ in grown simple families of increasing trees, can be written as indicators $B_{k} \stackrel{(d)}{=} \mathbb{I}\left(A_{k}\right)$, where $A_{k}$ denotes the event that node $k$ is on the path from the node $n$ to node $j$, which was shown in [16]. 
By using the decomposition of $\Delta_{n, j}$ in Lemma 3 we can simply multiply for $j+1 \leq$ $k \leq n-1$ the indicators $\mathbb{I}\left(A_{k}\right)$ with $k$ in order to obtain the following result .

Proposition 2. The weighted distance $\mathcal{W}_{n, j}$ between node $j$ and node $n$ in a size $n \geq j$ random grown simple increasing tree admits the following distribution law.

$$
\mathcal{W}_{n, j} \stackrel{(d)}{=}\left(\left(\mathcal{W}_{j+1, j}-(j+1)\right) \oplus \bigoplus_{k=j+1}^{n-1} \mathcal{B}_{k} \oplus n\right.
$$

where the random variables $\mathcal{B}_{k} \stackrel{(d)}{=} k \cdot \mathbb{I}\left(A_{k}\right)$ and $A_{k}$ denotes the event that node $k$ is on the path from node $n$ to node $j$. Further the $\mathcal{B}_{k}$ 's and $\mathcal{W}_{j+1, j}$ are independent.

Remark 5. Note that by directly translating Lemma 3 the weight of node $j+1$ is counted one time too many. This leads to the correcting term $j+1$.

We have not specified the the distribution law of $\mathcal{W}_{j+1, j}$ yet. It turned out in [16] that in most cases there is no simple description of $\Delta_{j+1, j}$, which can be used to determine the distribution of the weighted counterpart $\mathcal{W}_{j+1, j}$. For plane oriented trees we state the following result.

Proposition 3. For grown simple families of increasing trees with $c_{1}=-2 c_{2}$, the distribution law of $\mathcal{W}_{j+1, j}$ is given by

$$
\mathcal{W}_{j+1, j} \stackrel{(d)}{=}(2 j+1) \oplus \bigoplus_{k=1}^{j-1} \mathcal{C}_{k} \stackrel{(d)}{=}(2 j+1) \oplus \bigoplus_{k=1}^{j-1} k \cdot \mathbb{I}\left(\mathcal{A}_{k}\right)
$$

where $\mathcal{C}_{k}$ are a sequence of independent random variables such that

$$
\mathbb{P}\left(\mathcal{C}_{k}=k\right)=\frac{2}{2 k+1} \quad \text { and } \quad \mathbb{P}\left(\mathcal{C}_{k}=0\right)=1-\frac{2}{2 k+1} .
$$

We denote with $\mathcal{A}_{k}$ the event that node $k$ is on the path from node $j+1$ to node $j$. The indicator variables $\mathbb{I}\left(\mathcal{A}_{k}\right)$ 's are mutually independent.

Note that a decomposition of $\Delta_{j+1, j}$ of the form

$$
\Delta_{j+1, j} \stackrel{(d)}{=} 1 \oplus \sum_{k=1}^{j-1} \mathbb{I}\left(A_{k}\right)
$$


is possible for arbitrary grown simple families of increasing trees, but only in the case $c_{1}=-2 c_{2}$ the indicator variables are mutually independent. E.g. for recursive trees we get

$$
\begin{aligned}
\mathbb{P}\left\{A_{j-1}\right\} & =\mathbb{P}\left\{A_{j-1} \mid \Delta_{j, j-1}=1\right\} \mathbb{P}\left\{\Delta_{j, j-1}=1\right\}+\mathbb{P}\left\{A_{j-1} \mid \Delta_{j, j-1}>1\right\} \mathbb{P}\left\{\Delta_{j, j-1}>1\right\} \\
& =\frac{j-1}{(j-1) j}+\frac{j-2}{(j-1) j}=\frac{2 j-3}{j(j-1)} .
\end{aligned}
$$

Assuming that the $A_{k}$ 's are mutually independent we get further $\mathbb{P}\left\{A_{k}\right\}=\frac{2 k-1}{k(k+1)}$. But it can easily be seen that $\mathbb{P}\left\{A_{j-1}\right\} \mathbb{P}\left\{A_{j-2}\right\} \neq \mathbb{P}\left\{A_{j-1} A_{j-2}\right\}$, which leads to a contradiction. Hence for grown simple families of increasing trees with $c_{1} \neq-2 c_{2}$ we cannot specify the distribution of the weighted distance $\mathcal{W}_{j+1, j}$. However it is obvious that for fixed $j$ and $n \rightarrow \infty$ the contribution of $\mathcal{W}_{j+1, j}$ can be neglected, due to the fact that

$$
\mathcal{W}_{j+1, j} \leq 2 j+1+\sum_{k=1}^{j-1} k=\frac{j(j+3)}{2}+1
$$

For grown simple families of increasing trees admitting $c_{1}=-2 c_{2}$ we multiply the indicator functions $\mathbb{I}\left(A_{k}\right)$ with $k$, which gives Proposition 3.

\section{Deriving the limiting distribution}

\subsection{Weighted Depth}

First we present an extension of a result of Hwang and Tsai [8], p. 4, concerning the generalized Dickman distribution.

Lemma 4. The limiting distribution of the random variable

$$
X_{j}=\bigoplus_{k=1}^{j} \mathcal{B}_{k}\left(c_{1}, c_{2}\right),
$$

where the $\mathcal{B}_{k}$ 's are defined as in Proposition 1 , is for $j \rightarrow \infty$ asymptotically generalized Dickman $\mathrm{GD}(\theta)$ with parameter $\theta=1+\frac{c_{2}}{c_{1}}$. For $c_{1}=1$ and $c_{2}=0$ this just tells that the limiting distribution of $X_{j}$ is asymptotically Dickman:

$$
\lim _{j \rightarrow \infty} \mathbb{P}\left\{\frac{X_{j}}{j}<x\right\}=e^{-\gamma} \int_{0}^{x} \rho(v) d v, \quad(x>0) .
$$


The proof of Lemma 4 is completely analogous to the result of Hwang and Tsai $\left(c_{1}=1, c_{2}=0\right)$ in $[8]$.

We will follow the proof of Lemma 4 in [8] in order to prove Theorem 1. Let $\psi_{j}(t):=\mathbb{E}\left(e^{-t\left(W_{n, j}-j\right)}\right)$ denote the Laplace transform of the shifted random variable $W_{n, j}-j$. We have

$$
\phi_{j}(t)=\mathbb{E}\left(e^{-t\left(W_{n, j}-j\right)}\right)=\prod_{1 \leq k \leq j-1} \frac{k-1+e^{-t k}\left(1+\frac{c_{2}}{c_{1}}\right)}{k+\frac{c_{2}}{c_{1}}}=\prod_{1 \leq k \leq j-1}\left(1+\frac{\left(e^{-t k}-1\right)\left(1+\frac{c_{2}}{c_{1}}\right)}{k+\frac{c_{2}}{c_{1}}}\right) .
$$

It suffices, by Lévy's continuity theorem, and (2.3) to show that

$$
\lim _{j \rightarrow \infty} \phi_{j}\left(\frac{t}{j}\right)=\lim _{j \rightarrow \infty} \mathbb{E}\left(e^{-t \frac{\left(W_{n, j}-j\right)}{j}}\right)=\exp \left(\left(1+\frac{c_{2}}{c_{1}}\right) \int_{0}^{t} \frac{e^{-v}-1}{v} \mathrm{~d} v\right),
$$

for finite and real $t$. Now

$$
\begin{aligned}
\phi_{j}\left(\frac{t}{j}\right) & =\exp \left(\log \left(\phi_{j}\left(\frac{t}{j}\right)\right)\right)=\exp \left(\sum_{k=1}^{j-1} \log \left(1+\frac{\left(e^{-t k}-1\right)\left(1+\frac{c_{2}}{c_{1}}\right)}{k+\frac{c_{2}}{c_{1}}}\right)\right) \\
& =\exp \left(\left(1+\frac{c_{2}}{c_{1}}\right) \sum_{k=1}^{j-1} \frac{e^{\frac{-k t}{j}}-1}{k+\frac{c_{2}}{c_{1}}}+R_{j}(t)\right)
\end{aligned}
$$

where

$$
R_{j}(t):=\sum_{l \geq 2} \frac{(-1)^{l-1}}{l} \sum_{k=1}^{j-1} \frac{\left(\left(1+\frac{c_{2}}{c_{1}}\right)\right)^{l}\left(e^{\frac{-k t}{j}}-1\right)^{l}}{\left(k+\frac{c_{2}}{c_{1}}\right)^{l}}=\mathcal{O}\left(\frac{\left|t^{2}\right|}{j}\right) .
$$

Now we apply the Euler-MacLaurin summation formula to show

$$
\sum_{k=1}^{j-1} \frac{e^{\frac{-k t}{j}}-1}{k+\frac{c_{2}}{c_{1}}}=\int_{0}^{t} \frac{e^{-v}-1}{v} d v+\mathcal{O}\left(\frac{\left|t^{2}\right|}{j}\right) .
$$

\subsection{Weighted Distances}

We only sketch the proofs for the weighted distances since they are similar to the proof in Subsection 6.1. Let $\psi_{n}(t):=\mathbb{E}\left(e^{-t\left(\mathcal{W}_{n, j}-j-n\right)}\right)$ denote the Laplace transform of the shifted random variable $\mathcal{W}_{n, j}-j-n$.

$$
\begin{aligned}
\phi_{n}(t) & =\mathbb{E}\left(e^{-t\left(\mathcal{W}_{n, j}-j-n\right)}\right)=\mathbb{E}\left(e^{-t\left(\mathcal{W}_{j+1, j}-j\right)}\right) \prod_{j+1 \leq k \leq n-1} \frac{k-1+e^{-t k}\left(1+\frac{c_{2}}{c_{1}}\right)}{k+\frac{c_{2}}{c_{1}}} \\
& =\mathbb{E}\left(e^{-t\left(\mathcal{W}_{j+1, j}-j\right)}\right) \prod_{j+1 \leq k \leq n-1}\left(1+\frac{\left(e^{-t k}-1\right)\left(1+\frac{c_{2}}{c_{1}}\right)}{k+\frac{c_{2}}{c_{1}}}\right) .
\end{aligned}
$$


Hence

$$
\left.\phi_{n}\left(\frac{t}{n}\right)=\mathbb{E}\left(e^{-\frac{t\left(\mathcal{W}_{j+1, j}-j\right)}{n}}\right) \exp \left(\left(1+\frac{c_{2}}{c_{1}}\right) \sum_{k=j+1}^{n-1} \frac{\left(e^{-t k}-1\right)}{k+\frac{c_{2}}{c_{1}}}\right)+R_{n, j}(t)\right)
$$

where for fixed $j$ it can be seen that

$$
R_{n, j}(t):=\mathcal{O}\left(\frac{\left|t^{2}\right|}{n}\right)
$$

For fixed $j$ we use again Euler-MacLaurin summation to show that

$$
\sum_{k=j+1}^{n-1} \frac{\left(e^{-\frac{t k}{n}}-1\right)}{k+\frac{c_{2}}{c_{1}}}=\int_{0}^{t} \frac{e^{-v}-1}{v} \mathrm{~d} v+\mathcal{O}\left(\frac{\left|t^{2}\right|}{n}\right) .
$$

For grown simple families of increasing trees satisfying $c_{1}=-2 c_{2}$ we get

$$
\phi_{n}(t)=\mathbb{E}\left(e^{-t\left(\mathcal{W}_{n, j}-j-n\right)}\right)=\left(\prod_{1 \leq k \leq j-1}\left(1+\frac{\left(e^{-t k}-1\right)}{k+\frac{1}{2}}\right)\right)\left(\prod_{j+1 \leq k \leq n-1}\left(1+\frac{\left(e^{-t k}-1\right) \frac{1}{2}}{k-\frac{1}{2}}\right)\right)
$$

and further

$$
\phi_{n}\left(\frac{t}{n}\right)=\exp \left(\sum_{k=1}^{j-1} \frac{\left(e^{-\frac{t k}{n}}-1\right)}{k+\frac{c_{2}}{c_{1}}}+\frac{1}{2} \sum_{k=j+1}^{n-1} \frac{\left(e^{-\frac{t k}{n}}-1\right)}{k+\frac{c_{2}}{c_{1}}}+\hat{R}_{n, j}(t)+\tilde{R}_{n, j}(t)\right) .
$$

An application of Euler-MacLaurin summation then provides Theorem 3.

\section{Conclusion}

We presented the limit laws of weighted depths and distances in increasing trees. Note that it is possible to setup an even more general ( $f$-weighted) depth and distance by introducing a weight function $f(x)$ and defining

$$
W_{j, j}^{[f]} \stackrel{(d)}{=} f(j) \oplus \sum_{k=1}^{j-1} f(k) \cdot \mathbb{I}\left(A_{k}\right)
$$

where $A_{k}$ denotes the event that node $k$ is on the path from $j$ to the root. Furthermore

$$
\mathcal{W}_{n, j}^{[f]} \stackrel{(d)}{=} f(n) \oplus f(j) \oplus \sum_{k=1}^{j-1} f(k) \cdot \mathbb{I}\left(\mathcal{A}_{k}\right) \oplus \sum_{k=j+1}^{n-1} f(k) \cdot \mathbb{I}\left(\mathcal{A}_{k}\right),
$$


where for, $1 \leq k \leq j-1$ and $j+1 \leq k \leq n-1, \mathcal{A}_{k}$ denotes the event that node $k$ is on the path from $j$ to node $n$. E.g. $f(x) \equiv 1$ leads to the ordinary depth and distance (shifted by 1), where $f(x)=x$ leads to the weighted depth and weighted distance studied in this paper. Hence choosing $f(x)=x^{\alpha}$ would provide a natural transition between ordinary depths and weighted depths.

\section{Acknowledgements}

This work was supported by the Austrian Science Foundation FWF, grant S9608-N13. The authors thank an anonymous referee for many valuable comments.

\section{References}

[1] R. Aguech, N. Lasmar and H. Mahmoud, Extremal weighted path lengths in random binary search trees, Probability in the Engineering and Informational Sciences, to appear.

[2] F. Bergeron, P. Flajolet and B. Salvy, Varieties of increasing trees, Lecture Notes in Computer Science 581, 24-48, 1992.

[3] B. Bollobas and O. M. Riordan, Mathematical results on scale-free random graphs, in Handbook of graphs and networks, 1-34, Wiley-VCH, Weinheim, 2003.

[4] L. Devroye and R. Neininger, Distances and finger search in random binary search trees, SIAM Journal on Computing 33, 647-658, 2004.

[5] R. Dobrow, On the distribution of distances in recursive trees, Journal of Applied Probability 33, 749-757, 1996.

[6] R. Dobrow and R. Smythe, Poisson approximations for functionals of random trees, Random Structures \&f Algorithms 9, 79-92, 1996.

[7] R. Grübel and N. Stefanoski, Mixed Poisson approximation of node depth distributions in random binary search trees, Annals of Applied Probability 15, 279-297, 2005 .

[8] H. -K. Hwang and T. -H. Tsai, Quickselect and the Dickman function, Combinatorics, Probability and Computing, 11:4, 353-371, 2002. 
[9] M. Kuba, Analysis of node isolation procedures and label-based parameters in tree structures, PhD. thesis, TU Wien, 2006.

[10] M. Loève, Probability Theory I, 4th Edition, Springer-Verlag, New York, 1977.

[11] H. Mahmoud, Limiting distributions for path lengths in recursive trees, Probability in the Engineering and Informational Sciences 5, 53-59, 1991.

[12] H. Mahmoud, R. Modarres and R. Smythe, Analysis of QUICKSELECT: an algorithm for order statistics, RAIRO Theoretical Informatics and Applications 29, 255-276, 1995.

[13] H. Mahmoud and R. Smythe, On the distribution of leaves in rooted subtrees of recursive trees, Annals of Applied Probability 1, 406-418, 1991.

[14] H. Mahmoud and R. Smythe, A Survey of recursive trees, Theoretical Probability and Mathematical Statistics 51, 1-37, 1995.

[15] A. Meir and J. W. Moon, On the altitude of nodes in random trees, Canadian Journal of Mathematics 30, 997-1015, 1978.

[16] M. Kuba and A. Panholzer, On the distribution of distances between specified nodes in increasing trees, submitted.

[17] A. Panholzer and H. Prodinger, The level of nodes in increasing trees revisited, Random Structures \& Algorithms, to appear.

[18] M .D. Penrose and A. R. Wade, Random minimal directed spanning trees and Dickman-type distributions, Advances in Applied Probability 36, 691-714, 2004. 